\title{
A Temporal Noise Reduction Filter Based On Image Sensor Full-Frame Data
}

\author{
Angelo Bosco $^{1}$, Keith Findlater ${ }^{2}$, Sebastiano Battiato ${ }^{1}$, Alfio Castorina ${ }^{1}$ \\ ${ }^{1}$ Advanced System Technology Catania Lab - STMicroelectronics \\ ${ }^{2}$ Imaging Division, Edinburgh - STMicroelectronics \\ E-mail: \{Angelo.Bosco, Keith.Findlater, Sebastiano.Battiato, Alfio.Castorina\}@st.com
}

\begin{abstract}
This paper describes a temporal filter aimed to the simultaneous cancellation of fixed pattern noise and temporal noise from image sequences by exploiting all the data provided by a typical image sensor (e.g. CCD/CMOS).
\end{abstract}

\section{INTRODUCTION}

In order to improve the quality of images acquired by CCD/CMOS digital still cameras [1] we addressed the problem of filtering noise both spatially [2][3] and temporally [5][6]. Specifically, the noise reduction process was implemented directly in the Bayer Pattern domain [7]. In the temporal case, the execution of the temporal filter required the identification of flat areas in every frame in order determine the statistical properties of the superimposed noise. The estimation of the noise level was calculated over the current frame and it was used to filter the successive frame by means of the Duncan Filter [4][5][6]. In this paper we present a suitable technique able to realize a further enhancement. Image quality is improved by eliminating the Fixed Pattern Noise (FPN); avoiding the detection of flat areas further decreases processing time. Not all the data acquired by the imager is reproduced in the output color image; the presented method exploits the sensor non-image data for the estimation of noise levels.

\section{BASIC IDEA}

At the top of the image sensor, a sequence of black and dark lines is positioned (Figg.1, 2). Specifically, the black lines are not shielded from incident light, but they are held in minimum exposure, thus they contain very low noise; dark lines are shielded from incident light but they have the same exposure as the image lines. We aim to the simultaneous cancellation of FPN and temporal noise by using black and dark data respectively. By doing so, each frame can be filtered depending also on its own noise levels, instead of relying on the estimations computed over the previous frames.

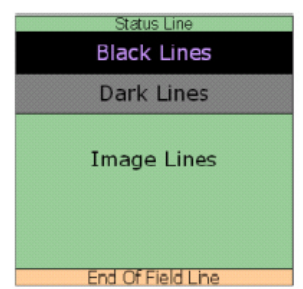

Figure 1 - Layout of the lines acquired by the image sensor.

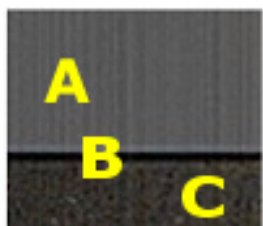

Figure 2: (A) Black lines contain information about fixed pattern noise. (B) In dark lines, pixel fluctuations are due to random noise thus they can be used to estimate the temporal noise variance $\sigma_{\mathrm{N}}$. (C) Image data.

\section{FILTERING PROCESS}

FPN Cancellation is achieved by continuously averaging the black sampled data, according to this scheme:

$F P N \_$Estimation $=F P N \_$Estimation -

$($ FPN_Estimation/LeakConstant $)+$

(FPN_CurrentSample/LeakConstant)

where:

FPN_Estimation: estimation of the FPN Signature

- FPN_CurrentSample: FPN signature, extracted from the current frame 
- LeakConstant: constant used in the integration process.

Pixel values must be scaled by taking into account the gains used when capturing frames. After some iterations, the FPN estimation converges towards the correct pixel values that must be subtracted, row after row, from the image data in order to get rid of the FPN. By changing the Leak Constant value and the number of black lines, the speed of convergence can be changed (see Fig.3). FPN is cancelled both from the image and the dark data. After that, the dark lines will only contain temporal noise. Illumination conditions change over time and adjustments in the gain values may generate adjacent frames having highly different noise variance values. This produces flickering in the filtered sequence that is eliminated by averaging the current noise estimation with the previous ones. After the estimation of the temporal noise variance $\sigma^{2}{ }_{N}$ on the dark lines, each pixel is filtered by using the Duncan Filter that selects, among three ranges centered at CurrentPixel, CurrentPixel $+\sigma_{\mathrm{N}}$ and CurrentPixel- $\sigma_{\mathrm{N}}$, the interval containing the maximum number of pixels; the range wideness $S$ depends on the standard deviation $\sigma_{\mathrm{N}}[4][5]$.

According to the selected range, a set of weigthing coefficients $w_{i}$ is chosen and the output is computed as:

$$
\text { FiltPix }=\frac{\sum_{i} w_{i}{ }^{*} \text { Pixel }_{i}}{\sum_{i} w_{i}}
$$

The proposed technique proves to be efficient especially in low light conditions where, due to the increased gains, FPN is more visible and temporal noise is more evident for the same reason. FPN cancellation can be switched on or off, depending on the gain used when capturing the image.

\section{EXPERIMENTAL RESULTS}

A series of experiments has been performed in order to find the suitable number of black lines needed to correctly estimate the fixed pattern noise (Fig.3). Similar tests have been performed on the dark lines used for the temporal noise level estimation.

The filtered sequences show improvements in terms of PSNR that depend on the particular illumination conditions of the acquired scene. Dim scenes suffer more noise than better illuminated ones. The number of black and dark lines has to be determined depending on the particular sensor in use.

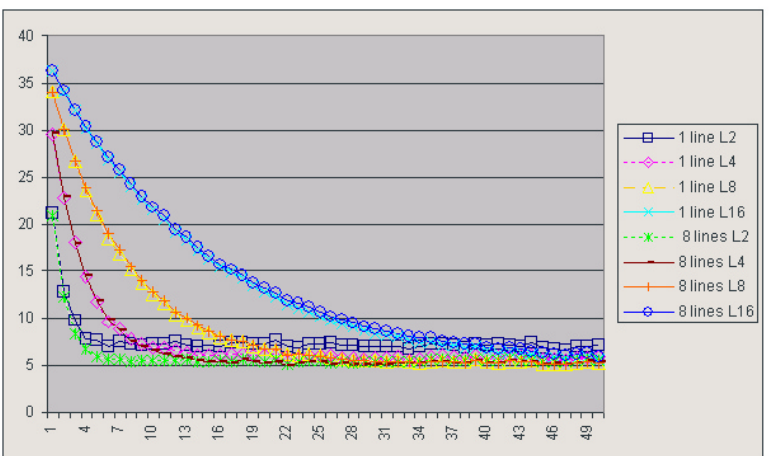

Figure 3 - The vertical axis represents the error in the estimation of the FPN signature; the horizontal axis represents the number of iterations needed until convergence is achieved. Each curve is obtained by changing the number of black lines and the Leak Constant L.

\section{REFERENCES}

[1] M. Mancuso, S. Battiato - "An Introduction to the Digital Still Camera Technology" - ST Journal of System Research. Vol.2, No.2, Dec.2001;

[2] A. Bosco, M. Mancuso - "Adaptive Filtering For Image Denoising" - In Proceedings of IEEE ICCE2001 (pp.208-209), Los Angeles, June 2001;

[3] A. Bosco - "Adaptive Image Denoising On Bayer Pattern" - ST Journal of System Research. Vol.2, No.2, Dec.2001;

[4] A. M. Borneo, L. Salinari, D. Sirtori, "An Innovative Adaptive Noise Reduction Filter for Moving Pictures Based on Modified Duncan Range Test", ST Journal of System Research, Vol.1, No.1, 2001;

[5] A. Bosco, M. Mancuso, S. Battiato, G. Spampinato, "Temporal Noise Reduction of Bayer Matrixed Video Data", In Proceedings of IEEE ICME'02 - International Conference on Multimedia and Expo 2002, pp.681-684, Lausanne, Switzerland, Aug. 2002;

[6] A. Bosco, M. Mancuso, S. Battiato, G. Spampinato, "Adaptive Temporal Filtering for CFA Video Sequences", In Proceedings of IEEE ACIVS'02 Advanced Concepts for Intelligent Vision Systems 2002, pp. 19-24, Ghent University, Belgium, Sept. 2002;

[7] B.E. Bayer, "Color Imaging Array", U.S. Patent 3,971,065-1976. 